\title{
Inkongruente Erwartungen an den Vorbereitungsdienst als Prädiktoren emotionaler Erschöpfung
}

\author{
Hendrik Lohse-Bossenz' , Juliane Rutsch², Birgit Spinath³ und Tobias Dörfler ${ }^{1}$ \\ 'Pädagogische Hochschule Heidelberg, Deutschland \\ ${ }^{2}$ Institut für Bildungsanalysen Baden-Württemberg, Deutschland \\ ${ }^{3}$ Ruprecht-Karls-Universität Heidelberg, Deutschland
}

\begin{abstract}
Zusammenfassung: Die vorliegende Studie untersucht die wahrgenommene Erfüllung von Erwartungen von Lehramtsanwärterinnen und -anwärtern an den Vorbereitungsdienst. Basierend auf empirischen Befunden aus der Arbeits- und Organisationspsychologie wird erwartet, dass nicht-erfüllte Erwartungen an den Vorbereitungsdienst mit einem Anstieg an emotionaler Erschöpfung assoziiert sein könnten. Es wurden 1109 Lehramtsanwärterinnen und -anwärter der Sekundarstufe I zu Beginn des Vorbereitungsdienstes (Erwartungen) und ein Jahr später (erfüllte Erwartungen) mit einer neu entwickelten Skala zu positiven („Nutzen“) und negativen („Kosten“) Wahrnehmungen des Vorbereitungsdienstes sowie ihrer emotionalen Erschöpfung befragt. Die Kosten-Nutzen-Skala konnte (erfüllte) Erwartungen an den Vorbereitungsdienst zu beiden Messzeitpunkten ausreichend gut erfassen. Auch die emotionale Erschöpfung wurde reliabel erfasst, wobei ein signifikanter Anstieg an emotionaler Erschöpfung im Untersuchungszeitraum festgestellt wurde. Response Surface Analysen wiesen auf einen Anstieg an emotionaler Erschöpfung im Untersuchungszeitraum hin, wenn Erwartungen an den Vorbereitungsdienst bezüglich dessen Kosten und Nutzen nicht mit der tatsächlichen Wahrnehmung übereinstimmten. Die Ergebnisse werden auf der Grundlage der aktuellen empirischen Befundlage diskutiert. Zuletzt wird ein Ausblick auf anschließende Forschungsarbeiten gegeben.
\end{abstract}

Schlüsselwörter: Response Surface Analyse, Vorbereitungsdienst, emotionale Erschöpfung, inkongruente Erwartungen, Lehramtsanwärterinnen und -anwärter

Not met expectations of pre-service teachers regarding the practical induction phase as predictors of emotional exhaustion

Abstract: The present study investigates the perceived fulfillment of expectations of pre-service teachers regarding the practical induction phase. Based on empirical findings from work and organizational psychology, it is expected that unfulfilled expectations of the induction phase might be associated with an increase in emotional exhaustion. A newly developed scale was used to survey 1109 pre-service secondary school teachers at the beginning (expectations) and one year later at the end of the induction phase (fulfilled expectations) about positive ("benefits") and negative ("costs") perceptions of the induction phase as well as pre-service teachers' emotional exhaustion. The cost-benefit scale was able to capture (fulfilled) expectations sufficiently well at both measurement time points. Emotional exhaustion was also reliably captured, with a significant increase in emotional exhaustion over the study period. Response surface analyses indicated an increase in emotional exhaustion over the study period when expectations regarding costs and benefits of the induction did not match actual perceptions. Results are discussed based on current empirical findings. Finally, an outlook on subsequent research is given.

Keywords: Response Surface Analysis, practical induction phase of pre-service teachers, emotional exhaustion, incongruent expectations, pre-service teachers

\section{Theoretischer Hintergrund}

\section{Einleitung}

Ziel des Vorbereitungsdienstes im Lehramt ist es, über das eigene unterrichtliche Handeln die bereits erworbenen Fähigkeiten und Fertigkeiten bedeutsam auf die spätere Berufspraxis hin zu entwickeln. Doch was erwarten angehende Lehrkräfte eigentlich von ihrer zweiten Phase der
Lehrerbildung - dem Vorbereitungsdienst, bei dem sie erstmals in substanziellem Umfang selbst unterrichten? Welchen Nutzen erwarten sie sich? Aber auch: Was muss investiert werden bzw. mit welchen (psychologischen) Kosten ist diese Phase der Lehrerbildung verbunden? Einhergehend damit stellt sich dann die Frage, wie angehende Lehrkräfte darauf reagieren, wenn sich der erwartete Nutzen nicht in der Form einstellt und die Kosten deutlich höher empfunden werden als ursprünglich angenommen. 
Die Forschung sowohl zur Passung von persönlichen Interessen zu Berufsfeldern (z.B. Kaub, Karbach, Spinath \& Brünken, 2016) als auch von Erwartungen an zukünftige Arbeitsstellen (z.B. Wanous, Poland, Premark \& Davis, 1992) legt nahe, dass eine Passung zwischen Merkmalen des Individuums (Interessenstruktur, Erwartungen an eine Arbeitsstelle) und Merkmalen der Arbeitsumgebung mit positiven Outcomes und eine fehlende Passung mit negativen Outcomes assoziiert ist.

Emotionale Erschöpfung wird im Rahmen der Lehrerbildung als ein negatives Outcome betrachtet und als eine Hauptkomponente von Burnout verstanden (z. B. Cropanzano, Rupp \& Byrne, 2003). Eine hohe emotionale Erschöpfung wirkt sich nicht nur unmittelbar negativ auf die Gesundheit der Betroffenen aus, beispielsweise durch psychosomatische Beschwerden wie Schlafstörungen, chronische Schmerzen ohne Befund oder funktionelle Herz-Kreislauf-Beschwerden (Bauer, Häfner, Kächele, Wirsching \& Dahlbender, 2003), sondern auch mittelbar auf die berufliche Leistung (z.B. Klusmann \& Richter, 2014; Pogere, Lopez-Sangil, Garcia-Senoran \& Gonzalez, 2019). Dabei steht das Ausmaß an emotionaler Erschöpfung von Lehrpersonen zum einen mit deren Verhalten im Unterricht (Klusmann, Kunter, Trautwein, Lüdtke \& Baumert, 2008) und zum anderen mit der Leistung und der Motivation der Schülerinnen und Schüler (Arens \& Morin, 2016; Klusmann \& Richter, 2014) in Zusammenhang. Demnach gilt es zu vermeiden, dass Lehrpersonen ein hohes Ausmaß an emotionaler Erschöpfung erleben. Da vor allem die Vielzahl an Belastungen in der Berufseinstiegsphase, den oftmals nur unzureichenden persönlichen und beruflichen Ressourcen der angehenden Lehrkräfte gegenübersteht (vgl. Cramer, Friedrich und Merk, 2018), zeigen sowohl nationale (Dicke et al., 2015; Dicke, Holzberger, Kunina-Habenicht, Linninger \& SchulzeStocker, 2016; Klusmann, Kunter, Voss \& Baumert, 2012) als auch internationale (u.a. Chang, 2009; Friedman, 2000; Harmsen, Helms-Lorenz, Maulana \& van Veen, 2018; Stokking, Leenders, Jong \& van Tartwijk, 2003) Studien eine bedeutsame Zunahme der emotionalen Erschöpfung als Folge beruflicher Beanspruchung.

Ob eine solche Zunahme an emotionaler Erschöpfung während des Vorbereitungsdienstes durch eine Inkongruenz zwischen Erwartungen an diese Phase und der dann tatsächlich wahrgenommenen Situation entsteht, ist Gegenstand dieser Studie. Sie nimmt Befunde aus der arbeitspsychologischen Forschung zu nicht-erfüllten Erwartungen zum Ausgangspunkt und prüft empirisch anhand von Response Surface Analysen, ob eine Inkongruenz von erwarteten und später wahrgenommenen Kosten bzw. Nutzen des Vorbereitungsdiensts mit einer stärkeren Zunahme emotionaler Erschöpfung zusammenhängen.

\section{Nicht-erfüllte Erwartungen an die Berufseinstiegsphase}

Porter und Steers (1973) haben postuliert, dass kongruente Erwartungen (met expectations) an eine Arbeitsstelle maßgeblich zur allgemeinen Arbeitszufriedenheit beitragen. Dabei wird herausgestellt, dass die Erwartungen an eine Arbeitsstelle individuell verschiedenen sind. Diese können verschiedene Bereiche der zukünftigen Arbeit betreffen: die konkreten Arbeitsinhalte, die Zusammenarbeit mit Kolleginnen und Kollegen sowie Vorgesetzten, Arbeitsbedingungen, die Arbeitsmenge oder die Arbeitszeiten. In verschiedenen Studien wurde untersucht, wie sich Erwartungen an eine Arbeitsstelle auf die berufliche Leistung und die Gesundheit von Arbeitnehmenden auswirken, wenn diese eintreffen (also kongruent sind) bzw. enttäuscht werden (also inkongruent sind). Anzunehmen ist, dass das Ausmaß der Passung zwischen den Erwartungen und den tatsächlichen Arbeitsbedingungen entscheidend für das Erleben ist. Je kongruenter Erwartungen und wahrgenommene Realität sind, desto zufriedener sollten Arbeitnehmende sein.

Wanous et al. (1992) zeigten in einer Meta-Analyse mit 31 Studien, dass kongruente Erwartungen mit der allgemeinen Arbeitszufriedenheit $(r=.39)$, dem Einsatz des Einzelnen für und in seiner Organisation $(r=.39)$, der Absicht dauerhaft im Unternehmen zu arbeiten $(r=.29)$, dem dauerhaften Verbleib an einer Arbeitsstelle $(r=.13)$ sowie der beruflichen Leistung $(r=.11)$ in Zusammenhang stehen. Für Mitarbeitende an Universitäten wurde festgestellt, dass kongruente Erwartungen unter anderem einen direkten Einfluss auf die Arbeitszufriedenheit haben (Ababneh, 2020).

Inkongruente Erwartungen drückten sich hingegen in negativer Art und Weise aus: Abgängerinnen und Abgänger einer Wirtschaftsschule wurden vor ihrem Abschluss sowie nach vier und zehn Monaten an ihrer neuen Arbeitsstelle zu ihren Erwartungen befragt. Inkongruente Erwartungen nach vier Monaten an der neuen Arbeitsstelle waren direkt mit der empfundenen Hilflosigkeit $(r=.23)$ sowie der Einarbeitung in die neue Arbeitsstelle $(r=-.22)$ assoziiert (Ashforth \& Saks, 2000). Technische Angestellte wurden vor ihrem ersten Arbeitstag sowie sechs Monate nach Beginn der Arbeitsstelle befragt: Mitarbeitende, die inkongruente Erwartungen aufwiesen - in dem Sinne, dass sie die Anforderungen an die neue Arbeitsstelle hinsichtlich Arbeitsumfang, Vereinbarkeit von Beruf und Familie sowie Zeitdruck unterschätzen - berichteten mehr Stresssymptome und eine geringere Arbeitszufriedenheit als Mitarbeitende mit kongruenten Erwartungen (Nelson \& Sutton, 1991). Dieses Ergebnis stützt die oben formulierte Annahme, dass inkongruente Erwartungen bedeutsam für die Entwicklung negativer Outcomes ist. Ok und Park 
(2018) zeigten darüber hinaus, dass inkongruente Erwartungen an eine Arbeitsstelle nach zwölf Monaten zu geringerer Arbeitszufriedenheit führten $(r=-.44, p<.001)$.

Zusammenfassend weist die Befundlage aus der Arbeitsund Organisationspsychologie darauf hin, dass inkongruente Erwartungen an eine Arbeitsstelle in verschiedenen Berufszweigen mit negativen Outcomes einhergehen können (Yu, 2014).

\section{Erwartungen an den Vorbereitungsdienst: Die empirische Befundlage}

Erwartungen an den Vorbereitungsdienst wurden bisher kaum untersucht. Munderloh (2018) weist darauf hin, dass Lehramtsanwärterinnen und -anwärter erwarten, dass in Seminarveranstaltungen im Rahmen des Vorbereitungsdienstes konkrete Handlungsweisen für das Unterrichten vermittelt würden. Diesem Wunsch kann aber nicht entsprochen werden, ,,weil der unterrichtliche Rahmen mit seinen moralischen, psychischen und sozialen Implikationen nur selektiv und damit verkürzend beachtet werden kann" (Görlich, 2004, S. 676). Dieses sei nach Görlich (2004) der Grund, warum die oftmals hohe Erwartungshaltung an die Übertragbarkeit von Wissen aus dem Studium in berufliche Handlungskompetenz im Rahmen der seminaristischen Ausbildung im Vorbereitungsdienst enttäuscht werde.

In Bezug auf Beratungsgespräche erwarten die Lehramtsanwärterinnen und -anwärter von ihren Mentorinnen und Mentoren, dass diese ihnen in konstruktiver Art und Weise Kritik geben, die zur weiteren professionellen Entwicklung anregen kann (Bovet \& Frommer, 2013).

Zuletzt hat eine Befragung der Lehramtsanwärterinnen und -anwärter ergeben (fünfstufige Skala mit $1=$ nicht $z u$ frieden, 5 = sehr zufrieden), dass die Zufriedenheit mit der seminaristischen Betreuung $\left(M_{\text {Baden-Württemberg }}=3.32, M_{\text {alle Bun- }}\right.$ $=3.70$ ) sowie der Offenheit und Transparenz der Leistungsanforderungen $\left(M_{\text {Baden-Wuirttemberg }}=2.58, M_{\text {alle Bundeslän- }}\right.$ $\left.{ }_{d e r}=3.18\right)$ im Land Baden-Württemberg unterdurchschnittlich ausgeprägt sind (Munderloh, 2018) und somit ausbaufähig erscheinen. Zusammenfassend zeigt sich, dass Erwartung an den Vorbereitungsdienst thematisiert, aber bislang nicht in einen systematischen Zusammenhang mit Outcomes gebracht werden.

\section{Entwicklung und Prädiktoren der emotionalen Erschöpfung im Vorbereitungsdienst}

Zur Entwicklung der emotionalen Erschöpfung als negative Folge beruflicher Beanspruchung bei Lehrpersonen liegt allgemein folgende Befundlage vor: Fives, Hamman und Olivarez (2007) berichten eine Abnahme der emotionalen Erschöpfung im Zeitraum von 12 Wochen während eines Praktikums während der Lehramtsausbildung. Im Rahmen dieser Studie wurden 49 Lehramtsstudierende in den USA zu drei Messzeitpunkten (zu Beginn des Praktikums, nach 4 bis 6 Wochen und nach 9 bis 11 Wochen) unter anderem zu ihrer emotionalen Erschöpfung befragt. Dagegen berichten Gavish und Friedman (2010) stabile Ausmaße an emotionaler Erschöpfung über die gesamte Berufskarriere hinweg. Hierzu legen Befunde nahe, dass die Ausprägungen an emotionaler Erschöpfung während der Berufseinstiegsphase der von erfahrenen Lehrkräften nach 20 Jahren Berufserfahrung entspricht (Klusmann et al., 2012). Goddard, O'Brien und Goddard (2006) konnten Anstiege der emotionalen Erschöpfung in den ersten beiden Berufsjahren zeigen. Klusmann et al. (2012) und Dicke et al. (2015) berichten für eine Stichprobe von Lehramtsanwärterinnen und -anwärtern in Deutschland einen signifikanten Anstieg der emotionalen Erschöpfung im ersten Jahr des Vorbereitungsdienstes. Voss und Kunter (2020) zeigen überdies einen umgekehrt u-förmigen Verlauf für die emotionale Erschöpfung im Vorbereitungsdienst: So stieg die emotionale Erschöpfung in einer Stichprobe von 163 Lehramtsanwärterinnen und -anwärtern mit dem Fach Mathematik im ersten Jahr des Vorbereitungsdienstes an und flachte im zweiten Jahr des Vorbereitungsdienstes wieder ab. Die Autorinnen hatten dies erwartet und so interpretiert, dass es sich zunächst um einen Praxisschock (Anstieg der emotionalen Erschöpfung im ersten Jahr des Vorbereitungsdienstes) mit anschließender Erholung (Abflachen der emotionalen Erschöpfung im zweiten Jahr des Vorbereitungsdienstes) handelte.

Die differenzielle Betrachtung der Entwicklung von emotionaler Erschöpfung zeigt jedoch, dass individuelle Unterschiede zwischen den Lehramtsanwärterinnen und -anwärtern vorliegen: Dicke et al. (2015) berichten bei $74 \%$ der angehenden Lehrpersonen eine stabile Ausprägung an emotionaler Erschöpfung, 23\% berichten einen Anstieg und nur 3\% einen Abfall im Zeitraum des ersten Jahres des Vorbereitungsdienstes.

Als Determinanten für differenzielle Entwicklungsverläufe der emotionalen Erschöpfung im Vorbereitungsdienst wurden Unterschiede in den Big Five, der Ausprägung an professionellen Wissensinhalten sowie strukturellen Bedingungen am Seminarstandort herausgestellt: In einer Untersuchung zu Prädiktoren der emotionalen Erschöpfung in der Berufseinstiegsphase von Lehrkräften berichten Klusmann et al. (2012), dass die Ausprägung der emotionalen Erschöpfung im Vorbereitungsdienst nach einem Schuljahr durch die Big Five Dimensionen Neurotizismus ( $\beta=.19$ ), Gewissenhaftigkeit ( $\beta=-.08)$ und Offenheit $(\beta=.10)$ sowie durch die Länge pädagogischer Vorerfahrungen $(\beta=-.08)$ und pädago- 
gisch-psychologisches Wissen $(\beta=-.08)$ signifikant vorhergesagt werden konnte $\left(R^{2}=.36\right)$. Voss, Wagner, Klusmann, Trautwein und Kunter (2017) untermauern diesen Befund, indem sie mithilfe von Latent-Change-Modellen zeigten, dass eine höhere Ausprägung der Big Five Dimension Neurotizismus höhere Ausprägungen an emotionaler Erschöpfung in zwei Kohorten von Lehramtsanwärterinnen und -anwärtern ( $\beta=-.66$ bzw. -.71$)$ vorhersagte. Daneben berichten Dicke et al. (2015) einen Zusammenhang zwischen dem Anstieg an emotionaler Erschöpfung und den professionellen Wissensinhalten learning and development und instruction: Je höher das Wissen zu Messzeitpunkt 1 ausgeprägt war, desto geringer war der Anstieg an emotionaler Erschöpfung im Untersuchungszeitraum. Zuletzt konnte gezeigt werden, dass eine als gut empfundene Betreuung durch die Mentorin bzw. den Mentor im Vorbereitungsdienst einen bedeutsamen Prädiktor für eine geringere Ausprägung an emotionaler Erschöpfung darstellte (Fives et al., 2007).

\section{Nicht-erfüllte Erwartungen an den Vorbereitungsdienst und Entwicklung emotionaler Erschöpfung}

Bisher fanden bestimmte Erwartungen von Lehramtsanwärterinnen und -anwärtern als mögliche Prädiktoren der emotionalen Erschöpfung im Vorbereitungsdienst kaum Berücksichtigung in empirischen Studien. Als eine der wenigen Studien untersuchte Keller-Schneider (2011) den Zusammenhang von erinnerten Berufswahlmotiven und dem Beanspruchungserleben in der Berufseinstiegsphase. Es konnte gezeigt werden, dass eine hohe Ausprägung sozialer Nützlichkeitsmotive mit einem erhöhten Beanspruchungserleben einhergehen. Da diese Studie nur querschnittlich angelegt war und die Berufswahlmotive retrospektiv erfasst wurden, sind die Effekte vergleichsweise gering und auch nicht als kausal zu interpretieren. Dennoch zeigt sich hieran, dass Erwartungen an den Beruf (in diesem Fall Berufswahlmotive) in einem Zusammenhang mit Professionalisierungsprozessen stehen können.

Möchte man spezifische Erwartungen an den Vorbereitungsdienst formulieren, so können diese den Nutzensaspekts im Sinne positiv konnotierter Erwartungen (vgl. „utility values“, Wigfield \& Eccles, 2000) betonen, wie beispielsweise die Erwartung das theoretisch erworbene Wissen im Lehramtsstudium nun praktisch anwenden zu können. Denkbar sind jedoch auch negativ konnotierte Erwartungen, die die einzubringenden persönlichen Kosten (vgl. ,emotional costs“, Wigfield \& Eccles, 2000) thematisieren, wie beispielsweise die Befürchtung einer hohen Prüfungslast. Hinsichtlich des Belastungserlebens ist anzunehmen, dass negative Erwartungen direkt zu einer stärkeren Belastung führen können, mit denen die jeweilige Person umzugehen hat. Es stellt sich jedoch auch die Frage, welche Effekte sich zeigen, wenn positive bzw. negative Erwartungen tatsächlich eintreffen (Kongruenz) oder nicht (Inkongruenz). Insbesondere Inkongruenzen sollten zu kognitiver Dissonanz (Festinger, 1957) der Lehramtsanwärterinnen und -anwärter führen, was einen Anstieg an emotionaler Erschöpfung bedingen könnte. Darüber hinaus ist anzunehmen, dass die Richtung der Inkongruenz einen Einfluss auf die Entwicklung der emotionalen Erschöpfung nehmen könnte. So ist zu erwarten, dass die Zunahme emotionaler Erschöpfung vergleichsweise höher ausfällt, wenn positive Erwartungen nicht erfüllt werden, als wenn diese übertroffen werden (Beispiel: „Ich kann das im Rahmen der ersten Phase der Lehrerbildung erworbene Fachwissen im Vorbereitungsdienst nicht in der Art und Weise in die Praxis umsetzen wie gehofft.“ vs. „Ich kann das im Rahmen der ersten Phase der Lehrerbildung erworbene Fachwissen besser im Vorbereitungsdienst nutzen, als ich erwartet habe."). Umgekehrt sollte es in Hinblick auf die Entwicklung emotionaler Erschöpfung eher von Vorteil sein, wenn negative Erwartungen unterschritten werden, als wenn diese übertroffen werden (Beispiel: „Ich empfinde die Prüfungslast im Vorbereitungsdienst weniger stark als erwartet.“ vs. „Die Prüfungslast im Vorbereitungsdienst ist stärker als ich erwartet habe.").

\section{Forschungsdesiderata}

Um die möglichen Auswirkungen zur Passung von Erwartungen an den Vorbereitungsdienst und der Wahrnehmung des Vorbereitungsdiensts auf die Entwicklung emotionaler Erschöpfung zu untersuchen, werden im Rahmen dieser Studie folgende Forschungsdesiderata bearbeitet: Erstens soll die bisher eingeschränkte Befundlage zu Erwartungen von Lehramtsanwärterinnen und -anwärtern an den Vorbereitungsdienst erweitert werden. Hierzu wird jeweils eine Skala zur Erfassung von positiven Erwartungen (erwarteter "Nutzen“ des Vorbereitungsdienstes, wie beispielsweise das Erlernen von praktisch bedeutsamen Verhaltensweisen) sowie negativen Erwartungen (erwartete „Kosten“ des Vorbereitungsdienstes, wie beispielsweise die Erwartung einer anstrengenden Zeit) entwickelt und bei Lehramtsanwärterinnen und -anwärtern eingesetzt.

Zweitens soll geprüft werden, ob es im Verlauf des ersten Jahres des Vorbereitungsdienstes zu einem Anstieg an emotionaler Erschöpfung der Lehramtsanwärterinnen und -anwärter kommt. Obwohl die empirische Befundlage hierzu nicht eindeutig ist (möglicherweise aufgrund variierender Lehrerbildungssysteme zwischen verschiedenen 
Ländern) wird basierend auf den Studien aus dem deutschsprachigen Raum angenommen, dass ein Anstieg an emotionaler Erschöpfung im ersten Jahr des Vorbereitungsdienstes verzeichnet werden kann. Beachtet werden muss hierbei jedoch, dass es differenzielle Effekte des Anstiegs an emotionaler Erschöpfung gibt, die im Folgenden beleuchtet werden sollen.

Drittens soll - basierend auf der empirischen Befundlage aus dem Fachbereich der Arbeits-und Organisationspsychologie - untersucht werden, ob inkongruente Erwartungen von Lehramtsanwärterinnen und -anwärtern an den Vorbereitungsdienst mit negativen Outcomes in Zusammenhang stehen. Als negatives Outcome wird der Anstieg an emotionaler Erschöpfung herangezogen, da es sich hierbei um eine gut beforschte Variable im Vorbereitungsdienst und dem Lehrerberuf handelt, welche mit unterrichtlichem Handeln in einem bedeutsamen Zusammenhang steht. Dabei sollen methodische Schwächen bisheriger Studien, die die Folgen inkongruenter Erwartungen untersuchten, durch den Einsatz von Response Surface Analysen (RSAs) überwunden werden. In Anlehnung an die Ergebnisse aus der Arbeits- und Organisationspsychologie ist zu erwarten, dass es zu einem Anstieg an emotionaler Erschöpfung kommen sollte, wenn inkongruente Erwartungen an den Vorbereitungsdienst vorliegen.

Um diese Forschungsdesiderata zu bearbeiten wurden folgende Hypothesen aufgestellt:

Hypothese 1: Positive („Nutzen“) und negative („Kosten“) Erwartungen an den Vorbereitungsdienst können als separate Dimensionen empirisch erfasst werden.

Hypothese 2: Es kommt zu einem Anstieg an emotionaler Erschöpfung im ersten Jahr des Vorbereitungsdiensts von Lehramtsanwärterinnen und-anwärtern.
Zu differenziellen Effekten der Entwicklung der emotionalen Erschöpfung im ersten Jahr des Vorbereitungsdienstes wurden folgende Hypothesen aufgestellt:

Hypothese 3: Wenn die eingetretenen Kosten die erwarteten Kosten übersteigen, kommt es zu einem Anstieg der emotionalen Erschöpfung.

Hypothese 4: Wenn der eingetretene Nutzen hinter dem erwarteten Nutzen zurückbleibt, kommt es zu einem Anstieg der emotionalen Erschöpfung.

\section{Methode}

\section{Stichprobe}

Diese Studie wurde im Rahmen des Forschungsprojekts "Effektive Kompetenzdiagnose in der Lehrerbildung“ (EKoL) durchgeführt (Rutsch, Rehm, Vogel, Seidenfuß \& Dörfler, 2018; Lohse-Bossenz, Rehm, Friesen, Seidenfuß, Rutsch, Vogel \& Dörfler, 2021). Hierin wurde eine Befragung von Lehramtsanwärterinnen und -anwärtern $\left(N=1109\right.$, Alter $_{\mathrm{MZP1}}: M=27.14, S D=4.01,71 \%$ weiblich $)$ an allen Staatlichen Seminaren für Didaktik und Lehrerbildung für die Sekundarstufe I an Werk-, Haupt- und Realschulen in Baden-Württemberg durchgeführt. Die teilnehmenden Lehramtsanwärterinnen und -anwärter wurden zu Beginn des Vorbereitungsdienstes (Februar 2017) und zehn bis zwölf Monate später (Dezember 2017 bis Februar 2018) im Rahmen einer Paper-Pencil-Erhebung befragt. Die Befragungen fanden jeweils in den Räumlichkeiten der Studienseminare statt. Die Teilnahme an der Befragung war freiwillig und dauerte $90 \mathrm{Mi}-$ nuten.

Tabelle 1. Itemformulierungen und Ladungen der Kosten-Nutzen-Skala zu den Messzeitpunkten 1 und 2

\begin{tabular}{|c|c|c|c|}
\hline \multicolumn{2}{|l|}{ Messzeitpunkt 1} & \multicolumn{2}{|l|}{ Messzeitpunkt 2} \\
\hline Itemformulierung & Ladung & Itemformulierung & Ladung \\
\hline $\begin{array}{l}\text { Der Vorbereitungsdienst wird eine anstrengende } \\
\text { Zeit. (K) }\end{array}$ & .77 & Der Vorbereitungsdienst ist eine anstrengende Zeit. (K) & .80 \\
\hline $\begin{array}{l}\text { Ich wäre froh. wenn ich den Vorbereitungsdienst } \\
\text { bereits hinter mir hätte. (K) }\end{array}$ & .78 & $\begin{array}{l}\text { Ich bin froh. wenn ich den Vorbereitungsdienst hinter mir } \\
\text { habe. (K) }\end{array}$ & .84 \\
\hline Ich fürchte die Prüfungslast im Vorbereitungsdienst. (K) & .47 & Die Prüfungslast im Vorbereitungsdienst war fürchterlich. (K) & .60 \\
\hline $\begin{array}{l}\text { Der Vorbereitungsdienst wird mich auf meine Tätigkeit } \\
\text { als Lehrkraft vorbereiten. }(\mathrm{N})\end{array}$ & .72 & $\begin{array}{l}\text { Der Vorbereitungsdienst hat mich auf meine Tätigkeit als } \\
\text { Lehrkraft vorbereitet. }(\mathrm{N})\end{array}$ & .75 \\
\hline Im Vorbereitungsdienst werde ich viel Neues lernen. (N) & 69 & Im Vorbereitungsdienst habe ich viel Neues gelernt. (N) & .70 \\
\hline $\begin{array}{l}\text { Im Vorbereitungsdienst kann ich viele praktische } \\
\text { Erfahrungen sammeln. die für meine berufliche } \\
\text { Zukunft wichtig sind. }(\mathrm{N})\end{array}$ & 69 & $\begin{array}{l}\text { Im Vorbereitungsdienst konnte ich viele praktische Erfahrungen } \\
\text { sammeln. die für meine berufliche Zukunft wichtig sind. }(\mathrm{N})\end{array}$ & 69 \\
\hline $\begin{array}{l}\text { Der Vorbereitungsdienst wird dazu beitragen, } \\
\text { dass ich ein guter Lehrer / eine gute Lehrerin werde. (N) }\end{array}$ & .57 & $\begin{array}{l}\text { Der Vorbereitungsdienst hat dazu beigetragen, dass ich ein guter } \\
\text { Lehrer/eine gute Lehrerin werde. (N) }\end{array}$ & .76 \\
\hline
\end{tabular}




\section{Verwendete Instrumente}

Zur Erfassung der Kosten und des Nutzens des Vorbereitungsdiensts aus Sicht der angehenden Lehrkräfte wurde eine zweidimensionale Skala entwickelt. Die Formulierungen der Items unterschieden sich zwischen MZP1 und MZP2 geringfügig, da zu MZP1 die erwarteten Kosten/der erwartete Nutzen und zu MZP2 der tatsächlich eingetretene Nutzen/die tatsächlich eingetretenen Kosten erfasst wurden (siehe Tabelle 1). Die Items waren auf einer fünfstufigen Skala $(1=$ Trifft gar nicht zu, $5=$ Trifft völlig zu) zu beantworten.

Zur Erfassung der emotionalen Erschöpfung wurden in Anlehnung an vorherige Studien (Dicke et al., 2015; Klusmann, Kunter, Trautwein, Lüdtke \& Baumert, 2008; Klusmann, Kunter, Voss \& Baumert, 2012; Klusmann, Kunter, Trautwein, Lüdtke \& Baumert, 2018) Items einer Selbstberichtsskala von Maslach, Jackson und Leiter (1996) eingesetzt. Die Items waren ebenfalls auf einer fünfstufigen Skala (1 = Trifft gar nicht $z u, 5=$ Trifft völlig zu) zu beantworten.

\section{Analysen}

Um die neu entwickelte Skala zu Erwartungen bezüglich der Kosten und des Nutzens des Vorbereitungsdienstes zu untersuchen, wird deren angenommene zweidimensionale Struktur mittels konfirmatorischer Faktorenanalysen geprüft, wobei das $R$-Paket lavaan (Rosseel, 2017) verwendet wird. Deskriptive Statistiken sowie psychometrische Gütekriterien, z.B. Reliabilitätsschätzungen, werden für die Kosten-Nutzen-Skala sowie die emotionale Erschöpfung für beide Messzeitpunkte berichtet. Außerdem wird geprüft, ob sich die beiden neu entwickelten Skalen empirisch von der zentralen Outcomevariablen emotionale Erschöpfung trennen lassen. Hierfür werden Modellvergleiche zwischen einem eindimensionalen Modell, bei dem alle Items auf einem gemeinsamen Faktor laden, und einem zweidimensionalen Modell mit beiden theoretisch angenommenen Faktoren gerechnet.

Um direkte Effekte von Erwartungen an den Vorbereitungsdienst auf die Entwicklung emotionaler Erschöpfung $\mathrm{zu}$ prüfen, wird in einem weiteren Schritt untersucht, ob die erwarteten Kosten und der erwartete Nutzen eine Veränderung emotionaler Erschöpfung im Verlauf des Vorbereitungsdienstes vorhersagen. Hierfür wird ein latentes lineares Strukturgleichungsmodell mit dem Kriterium emotionale Erschöpfung zu MZP2 und den Prädiktoren emotionale Erschöpfung zu MZP1, erwartete Kosten, erwarteter Nutzen sowie Geschlecht und Alter als Kontrollvariablen gerechnet.

Um in einem nächsten Schritt der zentralen Frage nach der Bedeutung inkongruenter Erwartungen auf die Entwicklung der emotionalen Erschöpfung im Vorbereitungsdienst nachzugehen, werden Response Surface Analysen (RSAs) durchgeführt (Box \& Draper, 1987; Schönbrodt, 2016; Schönbrodt, Humberg, Nestler \& Carlson, 2018; Wedderhoff, Chasiotis \& Rosman, 2020). Mithilfe von RSAs kann untersucht werden, ob die Übereinstimmung bzw. die Unterschiedlichkeit zweier Prädiktoren mit einem gemeinsamen Kriterium assoziiert ist, wobei diese Prüfung im dreidimensionalen Raum erfolgt. Die RSA ist der traditionellen Herangehensweise der Bildung von Differenzwerten und Korrelation mit einem Kriterium dahingehend überlegen, dass komplexere $\mathrm{Zu}$ sammenhänge zwischen Konstrukten geprüft werden können und Reliabilitätsprobleme, die mit der Bildung von Differenzwerten einhergehen, eingedämmt werden (z.B. Humberg, Nestler \& Back, 2018; Wedderhof, Chasiotis \& Rosman, 2020). Die Durchführung erfolgt mit Hilfe des R-Pakets RSA (Schönbrodt \& Humberg, 2020). Die Modellierung von RSAs basiert auf der Durchführung von polynomialen Regressionen (für mathematische Grundlagen siehe z.B. Edwards, 2007). Die allgemeine Gleichung um Zusammenhänge mittels polynomialer Regressionen zu beschreiben lautet $Z=b_{0}+b_{1} X+b_{2} Y+b_{3} X^{2}$ $+b_{4} X Y+b_{5} Y^{2}+e$. Dabei beschreibt $Z$ die abhängige Variable (hier: Anstieg der emotionalen Erschöpfung), $X$ (hier: erwartete / $\mathrm{r}$ Kosten / Nutzen) und $Y$ (hier: eingetretene $/ \mathrm{r}$ Kosten/ Nutzen) beschreiben die beiden Prädiktoren; d.h. die abhängige Variable regrediert auf die beiden Prädiktorvariablen $X$ und $Y$, die Interaktion zwischen beiden Prädiktorvariaben $X Y$ sowie die quadrierten Terme der beiden Prädiktorvariablen $X^{2}$ und $Y^{2}$. Zur Interpretation

Tabelle 2. Kennwerte der Kosten-Nutzen-Skala sowie der emotionalen Erschöpfung zu MZP1 und MZP2

\begin{tabular}{|c|c|c|c|c|c|c|c|}
\hline \multirow[t]{2}{*}{ Skala } & \multicolumn{3}{|c|}{ MZP1 } & \multicolumn{3}{|c|}{ MZP2 } & \multirow[b]{2}{*}{$d$} \\
\hline & $M$ & $S D$ & $\Omega$ & $M$ & $S D$ & $\Omega$ & \\
\hline Kosten & 4.17 & 0.66 & .65 & 4.26 & 0.73 & .74 & 0.13 \\
\hline Nutzen & 4.46 & 0.48 & .77 & 4.17 & 0.68 & .82 & 0.53 \\
\hline $\begin{array}{l}\text { Emotionale } \\
\text { Erschöpfung }\end{array}$ & 1.97 & 0.60 & .79 & 2.43 & 0.87 & .87 & 0.76 \\
\hline
\end{tabular}

Anmerkung: Alle Mittelwertvergleiche zwischen den Messzeitpunkten sind mit $p \leq .05$ signifikant. 
einer RSA wird das sog. „response surface pattern“ (Edwards, 1994; Harris, Ansaal \& Lievens, 2008) herangezogen - eine graphische Darstellung der Daten im dreidimensionalen Raum - wodurch die Interpretation der Ergebnisse erleichtert werden soll. Innerhalb dieses Ansatzes werden ausschließlich zwei Prädiktoren einbezogen. Durch die Hinzunahme weiterer Prädiktoren würde die Komplexität des Modells durch die Interaktionsterme sowie die quadratischen Effekte in nicht mehr zu interpretierender Weise zunehmen.

\section{Ergebnisse}

\section{Empirische Prüfung der Kosten-Nutzen- Skala}

Um die angenommene zweidimensionale Struktur der Kosten-Nutzen-Skala (Hypothese 1) zu überprüfen, wurde eine konfirmatorische Faktorenanalyse durchgeführt. Fehlende Werte wurden dabei über den Full Information Maximum Likelihood-Ansatz („FIML“) modellbasiert geschätzt (Lüdtke, Robitzsch, Trautwein \& Köller, 2007). Dieses zweidimensionale Modell wies zu MZP1 $\left(\chi^{2}(13)=\right.$ $36.67, p<.001$, RMSEA $=.04$, CFI $=.98)$ sowie MZP2 $\left(\chi^{2}(13)=70.15, p<.001\right.$, RMSEA $=.08$, CFI $\left.=.96\right)$ zufriedenstellende Fit-Indizes auf. Die Höhe der Faktorladungen zu MZP1 und MZP2 sind in Tabelle 1 dargestellt.

$\mathrm{Zu}$ MZP1 korrelierten die latenten Faktoren leicht positiv $(r=.11, p=.013)$; zu MZP2 standen diese nicht in statistisch bedeutsamem Zusammenhang $(r=-.01, p=.800$.). $\mathrm{Zu}$ beiden Messzeitpunkten war das zweidimensionale Modell einem alternativen eindimensionalen Modell überlegen (MZP1: $\Delta \chi^{2}(\Delta d f=1)=435.01, p<.001$, MZP2: $\left.\Delta \chi^{2}(\Delta d f=1)=569.48, p<.001\right)$. Die Skalen wiesen folgende Reliabilitäten auf: Kosten, $\Omega_{\mathrm{MZP1}}=.65, \Omega_{\mathrm{MZP} 2}=.74$; Nutzen, $\Omega_{\mathrm{MZP1}}=.77, \Omega_{\mathrm{MZP} 2}=.82$ (siehe auch Tabellen 2 und 3). Hypothese 1 kann damit verifiziert werden.

Des Weiteren sagten die erwarteten Kosten die eingetretenen Kosten vorher $(\beta=.41, p<.001)$ sowie den eingetretenen Nutzen $(\beta=.16, p=.031$.). Ferner sagte der erwartete Nutzen den eingetretenen Nutzen vorher $(\beta=.73$, $p<.001)$, nicht jedoch die eingetretenen Kosten $(\beta=.074$, $p=.229$; Modellfit: $\chi^{2}(72)=289.12, p<.001, \mathrm{RMSEA}=.05$, $\mathrm{CFI}=.93)$.

Um auszuschließend, dass die in den Kostenskalen eingesetzten Items in einem substantiellen Maße mit den Items zur emotionalen Erschöpfung überlappen, wurde die Trennbarkeit der beiden Konstrukte konfirmatorisch geprüft. Sowohl zu Messzeitpunkt $1\left(\Delta \chi^{2}(\Delta d f=1)=179.37\right.$, $p<.001)$ als auch Messzeitpunkt $2\left(\Delta \chi^{2}(\Delta d f=1)=181.79\right.$, $p<.001)$ zeigen die Modelle mit getrennten Dimensionen
Tabelle 3. Manifeste Korrelationen der Skalen Kosten, Nutzen und emotionale Erschöpfung zu MZP1 und MZP2

\begin{tabular}{lcclllll}
\hline & \multicolumn{2}{c}{ Kosten } & & \multicolumn{2}{c}{ Nutzen } \\
\cline { 1 - 2 } & MZP1 & MZP2 & & MZP1 & MZP2 \\
\hline Nutzen & .11 & -.01 & & & \\
Emotionale Erschöpfung & .33 & -.45 & & -.20 & -.23 \\
\hline
\end{tabular}

Anmerkung: Signifikante Korrelationen $p \leq .05$ sind fett markiert.

für Kosten und emotionale Erschöpfung im Vergleich zu den eindimensionalen Modellen eine bessere Passung zu den Daten. Die Korrelationen zwischen Kosten und emotionaler Erschöpfung liegen bei .38 ( $p \leq .001$, MZP1) sowie $.52(p \leq .001, \mathrm{MZP} 2)$.

\section{Entwicklung der emotionalen Erschöpfung im Vorbereitungsdienst}

Die emotionale Erschöpfung der Lehramtsanwärterinnen und -anwärter konnte über fünf Items zu beiden Messzeitpunkten mit zufriedenstellenden Modellfitindizes (MZP1: $\chi^{2}(10)=1002.56, p<.001$, RMSEA $=.09$, CFI $=.96 ;$ MZP2: $\chi^{2}(10)=1358.19, p<.001$, RMSEA $=.08$, CFI $=.98)$ sowie mit zufriedenstellenden Reliabilitäten $\left(\Omega_{\mathrm{MZP1}}=.79, \Omega_{\mathrm{MZP2}}=.87\right)$ abgebildet werden (siehe auch Tabellen 2 und 3).

Die emotionale Erschöpfung stieg im Untersuchungszeitraum signifikant an $\left(M_{\mathrm{MZP1}}=1.97, S D_{\mathrm{MZP} 1}=0.60 ; M_{\mathrm{MZP} 2}=\right.$ $\left.2.43, S D_{\mathrm{MZP2}}=0.87 ; t(651)=-15.30, p \leq .001\right)$. Es handelte sich mit $d=0.76$ um einen großen Effekt (Gignac \& Szodorai, 2016; Morris \& DeShon, 2002). Dieses Ergebnis repliziert vorherige Studien zum Anstieg der emotionalen Erschöpfung im Vorbereitungsdienst (z. B. Dicke et al., 2015). Die Datenlage unterstützt demnach Hypothese 2.

\section{Bedeutung von Erwartungen für die Entwicklung emotionaler Erschöpfung}

Um zu prüfen, ob sich die Erwartungen hinsichtlich des Nutzens bzw. der Kosten des Vorbereitungsdienstes auf die Entwicklung emotionaler Erschöpfung in direkter Weise auswirken, wurde eine latente Regression gerechnet, bei der die emotionale Erschöpfung zu Messzeitpunkt 1 sowie die erwarteten Kosten und der erwartete Nutzen als Prädiktoren die emotionale Erschöpfung zu Messzeitpunkt 2 vorhersagen. Zusätzlich wurden das Alter sowie das Geschlecht als weitere Kontrollvariablen aufgenommen. Neben dem autoregressiven Effekt von emotionaler Erschöpfung ( $\beta=.55, p \leq .001)$ zeigen sich weder für die Kosten $(\beta=.02, p=.647)$ noch für die Nutzensdimension 
( $\beta=.05, p=.231)$ signifikante Regressionskoeffizienten. Einzig das Alter zeigt einen kleinen Effekt $(\beta=.06, p \leq$ .001), dahingehend, dass ältere Personen eine stärkere Zunahme der emotionalen Erschöpfung im Verlauf des Vorbereitungsdienstes aufweisen.

\section{Response Surface Analysen}

Die bisherigen Ergebnisse weisen darauf hin, dass Erwartungen zu Kosten und Nutzen des Vorbereitungsdienstes ausreichend reliabel erfasst werden konnten. Weiterhin wurde ein signifikanter Anstieg der emotionalen Erschöpfung im Untersuchungszeitraum festgestellt. Außerdem konnten keine direkten Effekte der erwarteten Kosten und Nutzen des Vorbereitungsdiensts auf die Entwicklung emotionaler Erschöpfung bei den angehenden Lehrkräften gezeigt werden. Abschließend soll untersucht werden, ob eine Inkongruenz zwischen den Erwartungen an den Vorbereitungsdienst und der Realität des Vorbereitungsdienstes mit der Entwicklung der emotionalen Erschöpfung in Zusammenhang stehen.

Es wurde erwartet, dass eine Zunahme der emotionalen Erschöpfung insbesondere dann zu verzeichnen sein wird, wenn die tatsächlichen die erwarteten Kosten übersteigen oder wenn der tatsächliche Nutzen dem erwarteten Nutzen unterliegt. In beiden Fällen handelt es sich um Inkongruenzen zwischen Erwartungen und wahrgenommener Realität im Vorbereitungsdienst. Um die formulierten Annahmen zu prüfen, wurden RSAs durchgeführt, die explizit auf die Kongruenz bzw. Inkongruenz zweier Prädiktoren bei der Vorhersage einer dritten Variablen (Kriterium) abzielen. Als solches Kriterium wurde der Anstieg an emotionaler Erschöpfung als Differenzwert zwischen den Messzeitpunkten herangezogen (emotionale Erschöpfung $_{\mathrm{MZP2} 2}-$ emotionale Erschöpfung $\left._{\mathrm{MZP1}}\right)$. Hierbei drückt ein positiver Wert einen Anstieg an emotionaler Erschöpfung aus, ein negativer Wert ein Absinken.

\section{Kosten(in-)kongruenz und Zunahme emotionaler Erschöpfung}

Abbildung 1 zeigt den Response Surface Plot für den Anstieg an emotionaler Erschöpfung im Untersuchungszeitraum (senkrechte Achse) in Abhängigkeit der erwarteten (Kosten_1) und eingetretenen Kosten (Kosten_2). Die von vorn nach hinten verlaufende Diagonale entspricht dabei der sog. Kongruenzlinie, bei der die erwarteten Kosten mit den tatsächlichen Kosten direkt übereinstimmen. Bewegt man sich von dieser Linie nach links, liegen die tatsächlichen Kosten in einem höheren Bereich als die erwarteten

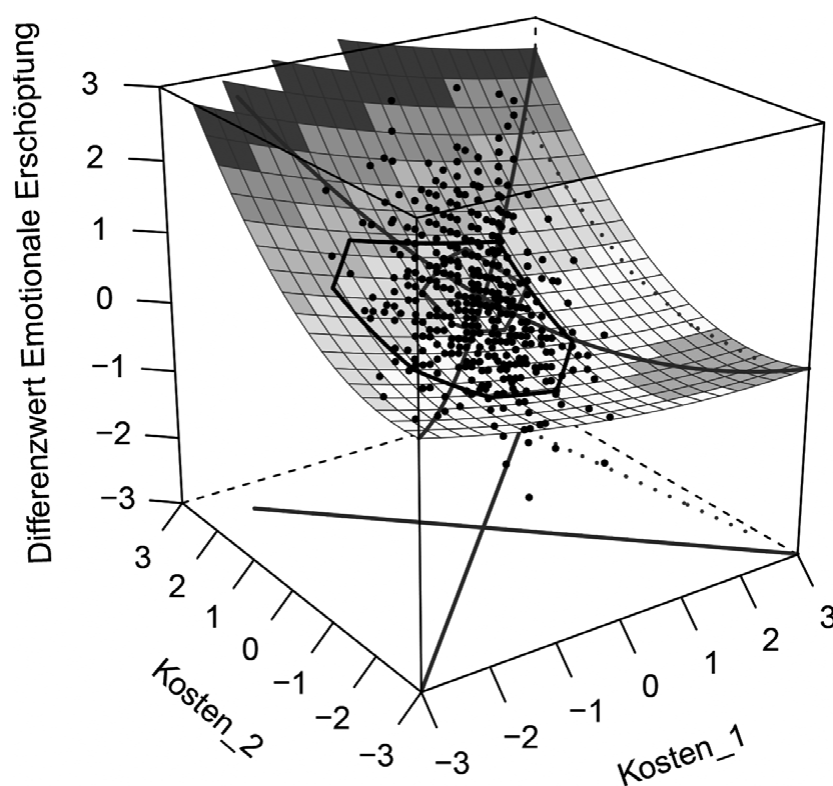

Abbildung 1. Die Abbildung zeigt den Response Surface Plot für die Zunahme Emotionaler Erschöpfung in Abhängigkeit von den erwarteten (Kosten_1) und eingetretenen (Kosten_2) Kosten. Die Variablen sind auf den Mittelwert zentriert. Die Linie mit Ursprung $X=-3$ und $Y=$ -3 stellt die sog. "line of congruence" dar und entspricht Fällen, in denen $X$ und $Y$ perfekt übereinstimmen. Die sog. , line of incongruence“ ist die dazu orthogonale Linie. Sie stellt diejenigen Fälle dar, bei denen Werte von $X$ eine negative Entsprechung von Y (Emotionale Erschöpfung) haben. Die Punktewolke stellt alle beobachteten Fälle dar. Diese sind auf der Ebene durch den sog. „,bagplot“ dargestellt, wobei der innere Kreis $50 \%$ der Fälle beinhaltet.

Kosten. Bewegt man sich nach rechts, liegen die tatsächlichen Kosten unterhalb der erwarteten Kosten.

Deskriptiv kann im Response Surface Plot abgelesen werden, dass es zu einem stärkeren Anstieg an emotionaler Erschöpfung kommt, wenn die eingetretenen Kosten die erwarteten Kosten übersteigen. Es ist auch zu erkennen, dass es zu einem geringfügigen Absinken bzw. zu einer Stabilisierung der emotionalen Erschöpfung kommt, wenn die eingetretenen Kosten niedriger sind als von den Lehramtsanwärterinnen und -anwärtern erwartet.

Um die statistische Signifikanz zu überprüfen, werden die RSA-Parameter ${ }^{1} a_{1}, a_{2}, a_{3}$ und $a_{4}$ herangezogen (siehe auch Bean, Forneris \& Brunet, 2016; Brunet, Gunell, Gaudreau \& Sabiston, 2015; Human, Dirks, DeLongis \& Chen, 2016; Shanock, Baran, Gentry, Pattison \& Heggestad, 2010): $a_{1}$ wird betrachtet um zu prüfen, ob es zu einem Anstieg bzw. Absinken der Outcomevariable kommt, wenn die Prädiktorvariablen ansteigen bzw. absinken; $\mathrm{a}_{2}$ wird betrachtet um zu prüfen, ob ein nicht-linearer Zusammenhang zwischen den Variablen vorliegt; $a_{3}$ wird betrachtet um die konkrete Richtung inkongruenter Erwartungen zu 
Tabelle 4. Übersicht über die Modellparameter der RSA zu erwarteten und eingetretenen Kosten

\begin{tabular}{|c|c|c|c|c|c|c|}
\hline Variable & Berechnung der Variable & $B$ & $S E$ & $p$ & $\begin{array}{c}\text { Untere Grenze } \\
\text { Konfidenzintervall }\end{array}$ & $\begin{array}{c}\text { Obere Grenze } \\
\text { Konfidenzintervall }\end{array}$ \\
\hline$b_{1}$ & Diff_EmEr Nutzen_1 & -0.18 & 0.06 & $<.001$ & -0.29 & -0.08 \\
\hline$b_{2}$ & Diff_EmEr Nutzen_2 & 0.55 & 0.05 & $<.001$ & 0.45 & 0.65 \\
\hline$b_{3}$ & Diff_EmEr Nutzen_12 & 0.02 & 0.05 & .637 & -0.07 & 0.12 \\
\hline$b_{4}$ & Diff_EmEr Nutzen_1_Nutzen_2 & -0.03 & 0.06 & .680 & -0.15 & 0.10 \\
\hline$b_{5}$ & Diff_EmEr Nutzen_22 & 0.12 & 0.03 & $<.001$ & 0.05 & 0.19 \\
\hline$b_{0}$ & Diff_EmEr 1 & 0.37 & 0.04 & $<.001$ & 0.30 & 0.45 \\
\hline a1 & $a 1=b 1+b 2$ & 0.37 & 0.06 & $<.001$ & 0.25 & 0.49 \\
\hline a2 & $a 2=b 3+b 4+b 5$ & 0.12 & 0.07 & .097 & -0.02 & 0.25 \\
\hline a3 & $a 3=b 1-b 2$ & -0.74 & 0.09 & $<.001$ & -0.91 & -0.57 \\
\hline a4 & $a 4=b 3-b 4+b 5$ & 0.17 & 0.10 & .101 & -0.03 & 0.37 \\
\hline$a_{5}$ & $a_{5}=b_{3}-b_{5}$ & -0.10 & 0.06 & .097 & -0.21 & 0.02 \\
\hline$x_{0}$ & $x_{0}=\left(b_{2}^{*} b_{4}-2 * b_{1} * b_{5}\right) /\left(4 * b_{3}^{*} b_{5}-b_{4}^{\wedge} 2\right)$ & 2.83 & 8.17 & .728 & -13.17 & 18.84 \\
\hline$Y_{0}$ & $Y_{0}=\left(b_{1} * b_{4}-2 * b_{2} * b_{3}\right) /\left(4 * b_{3} * b_{5}-b_{4}^{\wedge} 2\right)$ & -1.97 & 0.92 & .032 & -3.78 & -0.17 \\
\hline$p_{11}$ & $p_{11}=\left(b_{5}-b_{3}+\operatorname{sqrt}\left(\left(\left(b_{3}-b_{5}\right)^{\wedge} 2\right)+\left(b_{4}^{\wedge} 2\right)\right)\right) / b_{4}$ & -7.66 & 19.39 & .692 & -45.67 & 30.35 \\
\hline$p_{10}$ & $p_{10}:=Y_{0}-p_{11} * X_{0}$ & 19.73 & 108.64 & .855 & -193.19 & 232.66 \\
\hline$p_{21}$ & $p_{21}:=\left(b_{5}-b_{3}-\operatorname{sqrt}\left(\left(b_{3}-b_{5}\right)^{\wedge} 2+b_{4}^{\wedge} 2\right)\right) / b_{4}$ & 0.13 & 0.33 & .692 & -0.52 & 0.78 \\
\hline$p_{20}$ & $\mathrm{p}_{20}:=\mathrm{Y}_{0}-\mathrm{p}_{21} * \mathrm{X}_{0}$ & -2.34 & 0.53 & $<.001$ & -3.38 & -1.30 \\
\hline
\end{tabular}

Anmerkung: Modellparameter, die zur Beurteilung eines signifikanten Kongruenzeffekts herangezogen werden, sind fett markiert.

Tabelle 5. Übersicht über die Modellparameter der RSA zum erwarteten und eingetretenen Nutzen

\begin{tabular}{|c|c|c|c|c|c|c|}
\hline Variable & Berechnung der Variable & $B$ & $S E$ & $p$ & $\begin{array}{l}\text { Untere Grenze } \\
\text { Konfidenzintervall }\end{array}$ & $\begin{array}{c}\text { Obere Grenze } \\
\text { Konfidenzintervall }\end{array}$ \\
\hline$b_{1}$ & Diff_EmEr Nutzen_1 & 0.17 & 0.08 & .026 & 0.02 & 0.32 \\
\hline$b_{2}$ & Diff_EmEr Nutzen_2 & -0.29 & 0.07 & $<.001$ & -0.43 & -0.16 \\
\hline$b_{3}$ & Diff_EmEr Nutzen_12 & 0.27 & 0.13 & .043 & 0.01 & 0.53 \\
\hline$b_{4}$ & Diff_EmEr Nutzen_1_Nutzen_2 & -0.34 & 0.10 & .001 & -0.54 & -0.14 \\
\hline$b_{5}$ & Diff_EmEr Nutzen_22 & -0.01 & 0.05 & .824 & -0.11 & 0.09 \\
\hline$b_{0}$ & Diff_EmEr 1 & 0.38 & 0.04 & $<.001$ & 0.29 & 0.46 \\
\hline a1 & $a 1=b 1+b 2$ & -0.12 & 0.08 & .119 & -0.28 & 0.03 \\
\hline a2 & $\mathrm{a} 2=\mathrm{b} 3+\mathrm{b} 4+\mathrm{b} 5$ & -0.08 & 0.11 & .465 & -0.30 & 0.14 \\
\hline a3 & $\mathrm{a} 3=\mathrm{b} 1-\mathrm{b} 2$ & 0.46 & 0.12 & $<.001$ & 0.22 & 0.71 \\
\hline a4 & $a 4=b 3-b 4+b 5$ & 0.60 & 0.22 & .007 & 0.17 & 1.03 \\
\hline$a_{5}$ & $a_{5}=b_{3}-b_{5}$ & 0.28 & 0.14 & .050 & 0.00 & 0.56 \\
\hline$x_{0}$ & $x_{0}=\left(b_{2}^{*} b_{4}-2 * b_{1} * b_{5}\right) /\left(4^{*} b_{3}^{*} b_{5}-b_{4}^{\wedge} 2\right)$ & -0.81 & 0.30 & .006 & -1.39 & -0.23 \\
\hline$Y_{0}$ & $Y_{0}=\left(b_{1}{ }^{*} b_{4}-2 * b_{2} * b_{3}\right) /\left(4 * b_{3} * b_{5}-b_{4}^{\wedge} 2\right)$ & -0.79 & 0.50 & .115 & -1.76 & 0.19 \\
\hline$p_{11}$ & $p_{11}=\left(b_{5}-b_{3}+\operatorname{sqrt}\left(\left(\left(b_{3}-b_{5}\right)^{\wedge} 2\right)+\left(b_{4}^{\wedge} 2\right)\right)\right) / b_{4}$ & -0.47 & 0.14 & .001 & -0.75 & -0.20 \\
\hline$p_{10}$ & $p_{10}:=Y_{0}-p_{11} * X_{0}$ & -1.17 & 0.51 & .023 & -2.18 & -0.16 \\
\hline$p_{21}$ & $p_{21}:=\left(b_{5}-b_{3}-\operatorname{sqrt}\left(\left(b_{3}-b_{5}\right)^{\wedge} 2+b_{4}^{\wedge} 2\right)\right) / b_{4}$ & 2.12 & 0.63 & .001 & 0.88 & 3.37 \\
\hline$p_{20}$ & $\mathrm{p}_{20}:=\mathrm{Y}_{0}-\mathrm{p}_{21} * \mathrm{X}_{0}$ & 0.94 & 0.50 & .063 & -0.05 & 1.92 \\
\hline
\end{tabular}

Anmerkung: Modellparameter, die zur Beurteilung eines signifikanten Kongruenzeffekts herangezogen werden, sind fett markiert. 


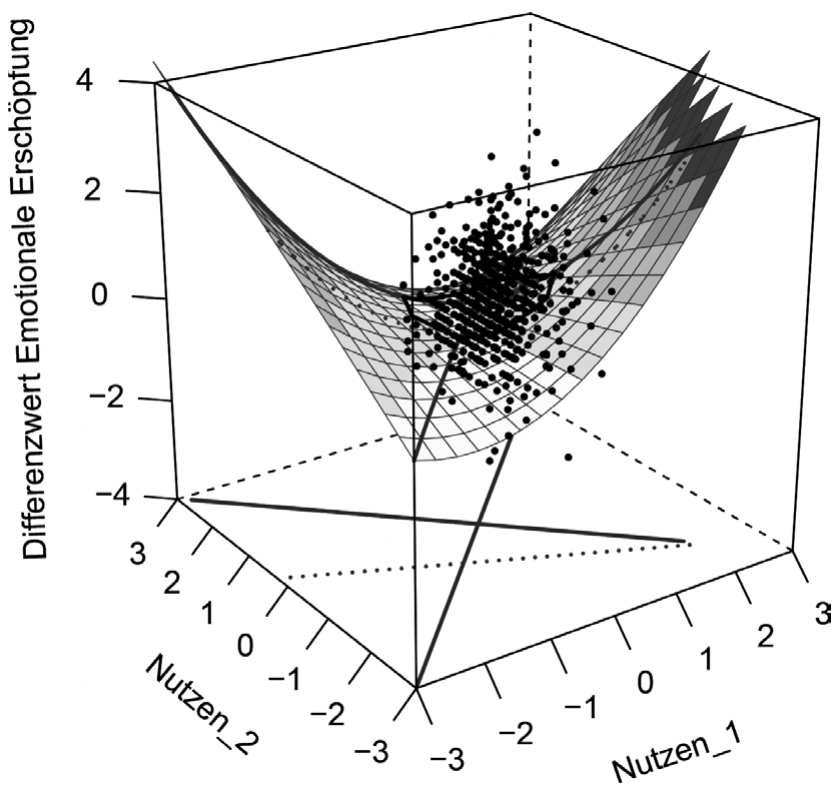

Abbildung 2. Die Abbildung zeigt den Response Surface Plot für die Zunahme Emotionaler Erschöpfung in Abhängigkeit vom erwarteten (Nutzen_1) und eingetretenen (Nutzen_2) Nutzen. Die Variablen sind auf den Mittelwert zentriert. Die Linie mit Ursprung $X=-3$ und $Y=-3$ stellt die sog. , ,line of congruence“ dar und entspricht Fällen, in denen $X$ und $Y$ perfekt übereinstimmen. Die Punktewolke stellt die beobachteten Fälle dar. Diese sind auf der Ebene durch den sog. „bagplot“ dargestellt, wobei der innere Kreis $50 \%$ der Fälle beinhaltet.

prüfen; $\mathrm{a}_{4}$ wird betrachtet um einen Inkongruenzeffekt unabhängig von der Richtung zu prüfen.

Hier liegen ein signifikant positives $a_{1}$, ein nicht signifikantes $a_{2}$, ein signifikant negatives $a_{3}$ und ein nicht signifikantes $\mathrm{a}_{4}$ vor (siehe Tabelle 4). Das bedeutet, der Anstieg an emotionaler Erschöpfung ist höher, wenn die Ausprägung an erwarteten und eingetretenen Kosten höher ist $\left(a_{1}\right)$, wobei ein linearerer Zusammenhang zwischen den Variablen vorliegt $\left(\mathrm{a}_{2}\right)$. Zudem kommt es zu einem Anstieg an emotionaler Erschöpfung, wenn die erwarteten Kosten die eingetretenen Kosten überschreiten und nicht andersherum $\left(\mathrm{a}_{3}, \mathrm{a}_{4}\right)$. Die Datenlage stützt demnach Hypothese 3.

\section{Nutzen(in-)kongruenz und Zunahme emotionaler Erschöpfung}

Abbildung 2 zeigt den Response Surface Plot für den Anstieg an emotionaler Erschöpfung im Untersuchungszeitraum in Abhängigkeit des erwarteten und eingetretenen Nutzens.

Es wurde erwartet, dass es zu einem Anstieg an emotionaler Erschöpfung kommt, wenn der eingetretene Nutzen (Nutzen_2) hinter dem erwarteten Nutzen (Nutzen_1) zurückbleibt. Deskriptiv kann im Response Surface Plot abgelesen werden, dass es zu einem Anstieg an emotionaler Erschöpfung kommt, wenn der eingetretene Nutzen hinter dem erwarteten Nutzen zurückbleibt, je- doch auch, wenn der eingetretene Nutzen den erwarteten Nutzen übersteigt.

Es liegen ein nicht signifikantes $a_{1}$, ein nicht signifikantes $a_{2}$, ein signifikant positives $a_{3}$ und ein positives $a_{4}$ vor (siehe Tabelle 5). Das bedeutet, der Anstieg an emotionaler Erschöpfung ist nicht höher bzw. niedriger, wenn die Ausprägung an erwarteten und eingetretenen Nutzen höher bzw. niedriger ist $\left(\mathrm{a}_{1}\right)$, wobei ein linearerer Zusammenhang zwischen den Variablen vorliegt $\left(\mathrm{a}_{2}\right)$. Zudem kommt es zu einem Anstieg an emotionaler Erschöpfung, wenn der eingetretene Nutzen hinter dem erwarteten Nutzen zurückbleibt und wenn der eingetretene Nutzen den erwarteten Nutzen übersteigt. $\left(a_{3}, a_{4}\right)$.

\section{Diskussion}

Beim Übergang von der ersten Phase der Lehrerbildung in die zweite Phase - den Vorbereitungsdienst - ändert sich das Anforderungsprofil für die angehenden Lehrkräfte in bedeutsamer Art und Weise. Viele gehen mit viel Zuversicht und Freude auf diese Phase der umfassenden Schulpraxis mit eigenverantwortlichem Unterricht zu: Viel Neues zu Lernen und Lernprozesse von Schülerinnen und Schülern zu gestalten, motivieren in besonderer Weise. Gleichzeitig ist den meisten angehenden Lehrkräften jedoch bewusst, dass die neuen Anforderungen die eigenen Ressourcen in besonderem Maße beanspruchen werden und der Vorbereitungsdienst somit auch einiges von ihnen fordern wird. Dass der Berufseinstieg mit einem erhöhten Belastungserleben einhergeht, konnte in verschiedenen Studien bereits gezeigt werden (z. B. Dicke et al., 2015).

Dass Erwartungen verhaltensrelevant sind, da sie u.a. zielgerichtetes Verhalten motivieren, ist empirisch auch für den schulischen Kontext (vgl. Wigfield \& Eccles, 2000) oder die Berufswahlentscheidungen von Lehrkräften (Watt \& Richardson, 2007) belegt. Die vorliegende Studie greift arbeitspsychologische Forschungsbefunde auf, die zeigen, dass die fehlende Erfüllung von Erwartungen an eine Arbeitsstelle mit negativen beruflichen Outcomes assoziiert ist (Wanous et al., 1992). Über die Untersuchung direkter Effekte von Erwartungen hinaus wird daher explizit geprüft, in welcher Weise eine Inkongruenz zwischen Erwartungen und (wahrgenommener) Realität mit der Veränderung emotionaler Erschöpfung als negatives Outcome des Vorbereitungsdienstes zusammenhängt. Es zeigen sich hierbei für die Stichprobe von angehenden Lehrkräften der Sekundarstufe 1 erwartungskonforme Effekte dahingehend, dass eine Unterschätzung der mit dem Vorbereitungsdienst einhergehenden Kosten mit einer stärkeren Zunahme emotionaler Erschöpfung zusammenhängt. Somit ist 
nicht nur die absolute Höhe der wahrgenommenen Kosten entscheidend, sondern eben auch die Diskrepanz zu den Erwartungen im Vorfeld. Hinsichtlich des Nutzens des Vorbereitungsdienstes zeigt sich, dass eine Inkongruenz zwischen Erwartungen und Realität mit einer $\mathrm{Zu}-$ nahme emotionaler Erschöpfung assoziiert ist. Dies entspricht einerseits den Annahmen, dass ein tatsächlicher Nutzen, der geringer ist als erwartet, zu Frustration und Resignation führt. Andererseits zeigt sich auch, dass ein Nutzen, der höher ist als vorher erwartet, auch mit einer stärkeren Zunahme emotionaler Erschöpfung zusammenhängt. Diesen erwartungswidrigen Befund gilt es in weiteren längsschnittlich angelegten Untersuchungen genauer in den Blick zu nehmen, um die dahinter liegenden Prozesse zu identifizieren.

\section{Kosten und Nutzen als Dimensionen von Erwartungen an den Vorbereitungsdienst}

Um Erwartungen von Lehramtsanwärterinnen und -anwärtern an den Vorbereitungsdienst zu erfassen, wurde eine Skala entwickelt, die positive („Nutzen“) sowie negative („Kosten“) Erwartungen beinhaltet. Diese beiden Dimensionen wurden aus der bisherigen (stark eingeschränkten) empirischen Befundlage abgeleitet und lassen sich innerhalb der Literatur den utility values (Wigfield \& Eccles, 2000) zuordnen. Während es darauf aufbauend für den Bereich der Berufswahlmotive entsprechende Operationalisierungen gibt (vgl. Watt \& Richardson, 2007), existierten für den Vorbereitungsdienst keine entsprechenden Instrumente.

Die empirischen Analysen zur Modellstruktur zeigten, dass sowohl erwartete und eingetretene Kosten als auch der erwartete und eingetretene Nutzen zufriedenstellend erfasst werden konnten (Hypothese 1). Hierbei ist anzumerken, dass die Reliabilitätsschätzung der Kosten-Skala zu MZP1 vergleichsweise gering ausfiel. Dies könnte auf die zum Zeitpunkt der Erhebung, nämlich zu Beginn des Vorbereitungsdienstes, noch diffusen Erwartungen an den Vorbereitungsdienst zurückzuführen sein. Eine einheitliche Repräsentation möglicher Kosten bzw. möglichen Nutzens des Vorbereitungsdienstes schien zu diesem Zeitpunkt bei den befragten Lehramtsanwärterinnen und -anwärtern noch nicht hinreichend ausgebildet gewesen zu sein. Für diese Vermutung spricht zudem die Beobachtung, dass die Reliabilitätsschätzungen der Kosten und der Nutzen-Skala zu MZP2 jeweils deutlich höher ausfallen. Weiterführende Analysen zu beiden Skalen werden klären müssen, ob andere Reliabilitätsschätzer zu anderen Befunden kommen. Beispielsweise kann eine hohe Retestreliabilität für die üblichen Zeiträume (bspw. 6 Wochen) durchaus angenommen werden.
Weiterführend ist anzumerken, dass die beiden Dimensionen „Kosten“ und „Nutzen“ die Erwartungen an den Vorbereitungsdienst nicht vollumfänglich abbilden können. Da eine eingeschränkte empirische Befundlage zu diesem Themenbereich vorgefunden wurde, erscheint es gewinnbringend, Erwartungen an den Vorbereitungsdienst breiter zu operationalisieren, als es in dieser Studie erfolgt ist (vgl. bspw. emotional costs, Wigfield \& Eccles, 2000)

\section{Nicht-erfüllte Erwartungen als Prädiktor emotionaler Erschöpfung}

Emotionale Erschöpfung als Folge kontinuierlicher Beanspruchung steht in Zusammenhang mit unterrichtlichem Handeln (Klusmann, Kunter, Trautwein, Lüdtke \& Baumert, 2008) und mit der Leistung und der Motivation der Schülerinnen und Schüler (Arens \& Morin, 2016; Klusmann \& Richter, 2014). Cramer und Kollegen (2018) skizzieren in ihrer Integration verschiedener Modelle der Belastungsforschung von Lehrkräften einen allgemeinen Wirkmechanismus, bei dem persönliche und berufliche Belastungen zu Beanspruchung führen können. In welchem Ausmaß dies geschieht, hängt von den individuellen Ressourcen einer Person ab.

Diverse empirische Studien konnten zeigen, dass emotionale Erschöpfung durch unterschiedlichste Merkmale vorhergesagt werden kann bzw. damit zumindest in $\mathrm{Zu}$ sammenhang steht (z.B. Voss, Wagner, Klusmann, Trautwein \& Kunter, 2017). Erwartungen an den Vorbereitungsdienst im Sinne von utility values (Wigfield \& Eccles, 2000) werden als ein weiteres Merkmal konzeptualisiert, welches einer kognitiven Repräsentation des Vorbereitungsdienstes entspricht. Hier zeigte sich kein bedeutsamer Einfluss auf die emotionale Erschöpfung. Aus der Analyse der Kongruenz-/Inkongruenz-Beziehungen von Erwartungen und Realität lassen sich demgegenüber jedoch bedeutsame $\mathrm{Zu}$ sammenhänge zur Entwicklung emotionaler Erschöpfung finden. Aus dieser Perspektive heraus ist nicht die Erwartung per se das relevante Merkmal, um den Umgang mit Belastungen zu beschreiben, sondern die Herstellung der Passung zwischen Erwartungen und Realität. Dass es sich hierbei um einen dynamischen und reziproken Prozess handelt, zeigen die Ergebnisse der Response Surface Analysen für die Nutzendimension. Hier ergibt sich ein weiterer Forschungsbedarf dahingehend, die Dynamiken zu beschreiben, um auf diese Weise auf die Genese emotionaler Erschöpfung einwirken zu können.

\section{Implikationen und Ausblick}

Die hier erzielten Ergebnisse sind für die Lehrerbildung in verschiedener Hinsicht relevant: Zunächst wurde ein Instru- 
ment entwickelt und längsschnittlich eingesetzt, welches Erwartungen an den Vorbereitungsdienst von Lehramtsanwärterinnen und -anwärtern ausreichend reliabel erfassen kann. Anschließende Studien sollten an diese erste Vorarbeit anknüpfen und dieses Instrument inhaltlich erweitern. Des Weiteren weisen die Ergebnisse dieser Studie auf einen Zusammenhang zwischen dem Anstieg an emotionaler Erschöpfung und inkongruenten Erwartungen an den Vorbereitungsdienst hin. Dies entspricht der Befundlage $\mathrm{zu}$ Erwartungen an die Berufseinstiegsphase in anderen Berufsfeldern. Hier gilt es weiterführende Untersuchungen anzuschließen, um die Auswirkungen von (inkongruenten) Erwartungen von angehenden Lehrpersonen in der Berufseinstiegsphase dezidierter zu beleuchten. Aus einer Forschungsperspektive heraus müssten die gefundenen Effekte über verschiedene Schulformen hinweg repliziert werden, da Belastungen schulformspezifisch auf Lehrkräfte wirken könnten. Weiterhin ist mit der emotionalen Erschöpfung ein Merkmal gewählt worden, dass in einem hohen Zusammenhang zur Kostendimension steht (MZP1: $r=.38, p \leq .001$; MZP2: $r=.52, p \leq .001)$. Hier scheint es von Interesse, positiv konnotierte Merkmale wie beispielsweise das Selbstwirksamkeitserleben oder das Engagement als Zielkriterium zu wählen. Aus einer praktischen Perspektive heraus verdeutlichen die Befunde die Notwendigkeit, neben unterrichtsbezogenen Aspekten auch andere Bereiche professioneller Kompetenz als Inhalte in der Praxisbegleitung von Studierenden zu betrachten (vgl. Korthagen \& Vasalos, 2005). So könnten bspw. ausgehend von Berufswahlmotiven spezifische mit dem Lehrberuf verbundene individuelle Erwartungen Gegenstand eines Reflexionsprozesses sein. Hierdurch können einerseits Erwartungen realistischer gestaltet werden oder andererseits die Wahrscheinlichkeit thematisiert werden, dass spezifische Erwartungen nicht erfüllt werden und wie damit aus einer individuellen Sicht umzugehen ist. Zuletzt zeigt sich in der Anwendung der Response Surface Analysen ein vielversprechender Ansatz, um Kongruenzund Inkongruenzhypothesen zu untersuchen. Unabhängig vom hier skizzierten Inhaltsbereich der Erwartungen an den Vorbereitungsdienst, sind Analysen denkbar, die beispielsweise die Perspektiven von Lehrkräften und deren Schülerinnen und Schüler in ein Verhältnis zueinander setzen.

\section{Literatur}

Ababneh, K. I. (2020). Effects of met expectations, trust, job satisfaction, and commitment on faculty turnover intentions in the United Arab Emirates (UAE). The International Journal of Human Resource Management, 31 (2), 303 - 334. https://doi.org/1 $0.1080 / 09585192.2016 .1255904$

Arens, A. K. \& Morin, A. J. S. (2016). Relations between teachers' emotional exhaustion and students' educational outcomes. Journal of Educational Psychology, 108 (6), 800 -813. https:// doi.org/10.1037/edu0000105
Ashforth, B. E. \& Saks, A. M. (2000). Personal control in organizations: A longitudinal investigation with newcomers. Human Relations, 53 (3), 311 -339. https://doi.org/10.1177/001872670 0533002

Bauer, J., Häfner, S., Kächele, H., Wirsching, M. \& Dahlbender, R. W. (2003). Burn-out und Wiedergewinnung seelischer Gesundheit am Arbeitsplatz. Psychotherapie Psychosomatik Medizinische Psychologie, 53, 213-222. https://doi.org/10.1055/s-200338865

Bean, C., Forneris, T. \& Brunet, J. (2016). Investigating discrepancies in program quality related to youth volleyball athletes' needs support. Psychology of Sport and Exercise, 26, 154-163. https://doi.org/10.1016/j.psychsport.2016.07.001

Bovet, G. \& Frommer, H. (2013). Praxis Lehrerberatung - Lehrerbeurteilung. Konzepte für Ausbildung und Schulaufsicht. Baltmannsweiler: Schneider-Verl. Hohengehren.

Box, G. E. P. \& Draper, N. R. (1987). Empirical model-building and response surfaces. New York: Wiley.

Brunet, J., Gunnell, K. E., Gaudreau, P. \& Sabiston, C. M. (2015). An integrative analytical framework for understanding the effects of autonomous and controlled motivation. Personality and Individual Differences, 84, 2-15. https://doi.org/10.1016/j.paid. 2015.02.034

Chang, M.-L. (2009). An appraisal perspective of teacher burnout: Examining the emotional work of Teachers. Educational Psychology Review, 21 (3), 193 -218. https://doi.org/10.1007/s10648009-9106-y

Cramer, C., Friedrich, A. \& Merk, S. (2018). Belastung und Beanspruchung im Lehrerinnen- und Lehrerberuf: Übersicht zu Theorien, Variablen und Ergebnissen in einem integrativen Rahmenmodell. Bildungsforschung, 1, 1-23. https://doi.org/10.25539/ bildungsforschun.v0i1.237

Cropanzano, R., Rupp, D. E. \& Byrne, Z. S. (2003). The relationship of emotional exhaustion to work attitudes, job performance, and organizational citizenship behaviors. Journal of Applied Psychology, 88 (1), 160 - 169. https://doi.org/10.1037/0021-9010. 88.1 .160

Dicke, T., Holzberger, D., Kunina-Habenicht, O., Linninger, C. \& Schulze-Stocker, F. (2016). „Doppelter Praxisschock“ auf dem Weg ins Lehramt? Verlauf und potenzielle Einflussfaktoren emotionaler Erschöpfung während des Vorbereitungsdienstes und nach dem Berufseintritt. Psychologie in Erziehung und Unterricht, 63 (4), 244 - 257. https://doi.org/10.2378/peu2016.art20d

Dicke, T., Parker, P. D., Holzberger, D., Kunina-Habenicht, O., Kunter, M. \& Leutner, D. (2015). Beginning teachers' efficacy and emotional exhaustion. Latent changes, reciprocity, and the influence of professional knowledge. Contemporary Educational Psychology, 41, 62 - 72. https://doi.org/10.1016/j.cedpsych.2014.11.003

Edwards, J. R. (1994). The study of congruence in organizational behavior research: Critique and proposed alternative. Organizational Behavior and Human Decision Processes, 58, 51-100. https://doi.org/10.1006/obhd.1994.1029

Edwards, J. R. (2007). Polynomial regression and response surface methodology. In C. Ostroff \& T. A. Judge (Hrsg.), Perspectives on Organizational Fit (S.361 - 372). San Francisco: Jossey-Bass.

Festinger, L. (1957). A Theory of cognitive dissonance. Stanford, CA: Stanford University Press.

Fives, H., Hamman, D. \& Olivarez, A. (2007). Does burnout begin with student-teaching? Analyzing efficacy, burnout, and support during the student-teaching semester. Teaching and Teacher Education, 23 (6), 916 - 934. https://doi.org/10.1016/j.tate.2006. 03.013

Friedman, I. A. (2000). Burnout in teachers: Shattered dreams of impeccable professional performance. Journal of Clinical Psychology, 56 (5), 595 -606. https://doi.org/10.1002/(SICI)10974679(200005)56:5<595::AID-JCLP2>3.0.CO;2-Q 
Gavish, B. \& Friedman, I. A. (2010). Novice teachers' experience of teaching: a dynamic aspect of burnout. Social Psychology of Education, 13 (2), $141-167$.

Gignac, G. E. \& Szodorai, E. T. (2016). Effect size guidelines for individual differences researchers. Personality and Individual Differences, 102, 74-78. https://doi.org/10.1016/j.paid.2016.06. 069

Goddard, R., O'Brien, P. \& Goddard, M. (2006). Work environment predictors of beginning teacher burnout. British Educational Research Journal, 32 (6), 857 - 874.

Görlich, H. (2004). Lehrerbildung zwischen Hochstapelei und irritierender Praxis. Ein Beitrag zur Enttabuisierung pädagogischen und didaktischen Bemühens aus der Sicht eines Seminardirektors. Pädagogische Rundschau, 58 (6), 653 - 684.

Harmsen, R., Helms-Lorenz, M., Maulana, R. \& van Veen, K. (2019). The longitudinal effects of induction on beginning teachers' stress. The British Journal of Educational Psychology, 89 (2), 259 - 287. https://doi.org/10.1111/bjep.12238

Harris, M. M., Ansaal, F. \& Lievens, F. (2008). Keeping up with the Joneses: A field study of the relationships among upward, lateral, and downward comparisons and pay level satisfaction. Journal of Applied Psychology, 93, 665-673. https://doi.org/ 10.1037/0021-9010.93.3.665

Human, L. J., Dirks, M. A., DeLongis, A. \& Chen, E. (2016). Congruence and incongruence in adolescents' and parents' perceptions of the family: Using Response Surface Analysis to examine links with adolescents' psychological adjustment. Journal of Youth and Adolescence, 45 (10), 2022-2035. https://doi. org/10.1007/s10964-016-0517-z

Humberg, S., Nestler, S. \& Back, M. D. (2018). Response surface analysis in personality and social psychology. Social Psychological and Personality Science, 1-11. https://doi.org/10.1177/1948550618 757600

Kaub, K., Karbach, J., Spinath, F. M. \& Brünken, R. (2016). Person-job fit in the field of teacher education - An analysis of vocational interests and requirements among novice and professional science and language teachers. Teaching and Teacher Education, 55, 217-227. https://doi.org/10.1016/j.tate.2016.01.010

Keller-Schneider, M. (2011). Die Bedeutung von Berufswahlmotiven von Lehrpersonen in der Bewältigung beruflicher Anforderungen in der Berufseingangsphase. Lehrerbildung auf dem Prüfstand, 4 (2), $157-185$.

Klusmann, U., Kunter, M., Trautwein, U., Lüdtke, O. \& Baumert, J. (2008). Teachers' occupational well-being and quality of instruction. The important role of self-regulatory patterns. Journal of Educational Psychology, 100 (3), 702 - 715. https://doi.org/10. 1037/0022-0663.100.3.702

Klusmann, U., Kunter, M., Voss, T. \& Baumert, J. (2012). Berufliche Beanspruchung angehender Lehrkräfte: Die Effekte von Persönlichkeit, pädagogischer Vorerfahrung und professioneller Kompetenz. Zeitschrift für Pädagogische Psychologie, 26 (4), 275-290. https://doi.org/10.1024/1010-0652/a000078

Klusmann, U. \& Richter, D. (2014). Beanspruchungserleben von Lehrkräften und Schülerleistung. Zeitschrift für Pädagogik, 60 (2), $202-224$.

Klusmann, U., Kunter, M., Trautwein, U., Lüdtke, O. \& Baumert, J. (2008). Engagement and emotional exhaustion in teachers. Does the school context make a difference? Applied Psychology, 57, 127 -151. https://doi.org/10.1111/j.1464-0597.2008.00358.x

Korthagen, F. \& Vasalos, A. (2005). Levels in reflection: core reflection as a means to enhance professional growth. Teachers and Teaching: theory and practice, 11 (1), $47-71$. https://doi.org/ 10.1080/1354060042000337093

Lohse-Bossenz, H., Rehm, M., Friesen, M., Seidenfuß, M., Rutsch, J., Vogel, M. \& Dörfler, T. (Hrsg.). (2021). Professionalisierung in der Lehrerbildung. Erkenntnisse und Perspektiven des inter- disziplinären Forschungsprogramms „Effektive Kompetenzdiagnose in der Lehrerbildung“. Münster: Waxmann.

Lüdtke, O., Robitzsch, A., Trautwein, U. \& Köller, O. (2007). Umgang mit fehlenden Werten in der psychologischen Forschung. Psychologische Rundschau, 58 (2), 103-117. https://doi.org/10. 1026/0033-3042.58.2.103

Maslach, C., Jackson, S. E. \& Leiter, M. P. (1996). Maslach Burnout Inventory Manual (3. Aufl.). Palo Alto, CA: Consulting Psychologists Press.

Morris, S. B. \& DeShon, R. P. (2002). Combining effect size estimates in meta-analysis with repeated measures and independentgroups designs. Psychological Methods, 7 (1), 105 -125. https:// doi.org/10.1037/1082-989x.7.1.105

Munderloh, O. (2018). Das Referendariat aus der Sicht der Referendar/innen (1. Auflage). Weinheim: Beltz Juventa.

Nelson, D. L. \& Sutton, C. D. (1991). The relationship between newcomer expectations of job stressors and adjustment to the new job. Work \& Stress, 5 (3), 241 -251. https://doi.org/10.1080/ 02678379108257022

Ok, C. \& Park, J. (2018). Change in newcomers' job satisfaction: Met-expectations effect as a moderator. Social Behavior and Personality: an international journal, 46 (9), 1513 - 1521. https:// doi.org/10.2224/sbp.6843

Pogere, E. F., López-Sangil, M. C., García-Señorán, M. M. \& González, A. (2019). Teachers' job stressors and coping strategies: Their structural relationships with emotional exhaustion and autonomy support. Teaching and Teacher Education, 85, 269 - 280. https://doi.org/10.1016/j.tate.2019.07.001

Porter, L. W. \& Steers, M. S. (1973). Organizational, work, and personal facotos in employee turnover and absenteeism, 80 (2), 151 - 176. https://doi.org/10.1037/h0034829

Rosseel, Y. (2017). Package 'lavaan'. Zugriff am 06.03.2017. Verfügbar unter https://cran.r-project.org/web/packages/lavaan/lava an.pdf

Rutsch, J., Rehm, M., Vogel, M., Seidenfuß, M. \& Dörfler, T. (Hrsg.). (2018). Effektive Kompetenzdiagnose in der Lehrerbildung. Professionalisierungsprozesse angehender Lehrkräfte untersuchen. Wiesbaden: Springer. https://doi.org/10.1007/978-3-658-20121-0

Schönbrodt, F. D. (2016). Testing fit patterns with polynomial regression models. Verfügbar unter osf.io/3889z

Schönbrodt, F. D., Humberg, S., Nestler, S. \& Carlson, E. (2018). Testing similarity effects with dyadic Response Surface Analysis. European Journal of Personality, 32 (6), 627 -641. https:// doi.org/10.1002/per.2169

Stokking, K., Leenders, F., de Jong, J. \& van Tartwijk, J. (2003). From student to teacher: reducing practice shock and early dropout in the teaching profession. European Journal of Teacher Education, 26 (3), 329 - 350. https://doi.org/10.1080/0261976032000 128175

Schönbrodt, F. D. \& Humberg, S. (2020). Package „RSA“. Verfügbar unter https://cran.r-project.org/web/packages/RSA/RSA.pdf

Shanock, L. R., Baran, B. E., Gentry, W. A., Pattison, S. C. \& Heggestad, E. D. (2010). Polynomial regression with Response Surface Analysis: A powerful approach for examining moderation and overcoming limitations of difference scores. Journal of Business and Psychology, 25 (4), 543-554. https://doi.org/10.1007/s10869010-9183-4

Voss, T. \& Kunter, M. (2020). "Reality Shock" of Beginning Teachers? Changes in Teacher Candidates' Emotional Exhaustion and Constructivist-Oriented Beliefs. Journal of Teacher Education, 71 (3), 292 - 306. https://doi.org/10.1177/0022487119839700

Voss, T., Wagner, W., Klusmann, U., Trautwein, U. \& Kunter, M. (2017). Changes in beginning teachers' classroom management knowledge and emotional exhaustion during the induction phase. Contemporary Educational Psychology, 51, 170-184. https://doi.org/10.1016/j.cedpsych.2017.08.002 
Wanous, J. P., Poland, T. P., Premark, S. L. \& Davis, K. S. (1992). The effects of met expectations on newcomer attitudes and behaviors. Journal of Applied Psychology, 77 (3), 288 - 297. https://doi. org/10.1037/0021-9010.77.3.288

Watt, H. M. G. \& Richardson, P. W. (2007). Motivational factors influencing teaching as a career choice: Development and validation of the FIT-Choice scale. Journal of Experimental Education, 75 (3), 167 - 202. https://doi.org/10.3200/JEXE.75.3.167-202

Wedderhoff, O., Chasiotis, A. \& Rosman, T. (2020). Erkenne dich selbst! Psychologische Rundschau, 71 (2), 134 -142. https://doi. org/10.1026/0033-3042/a000486

Wigfield \& Eccles. (2000). Expectancy-Value Theory of Achievement Motivation. Contemporary Educational Psychology, 25 (1), 68 - 81. https://doi.org/10.1006/ceps.1999.1015

Yu, K. Y.T. (2014). Person-organization fit effects on organizational attraction: A test of an expectations-based model. Organizational Behavior and Human Decision Processes, 124 (1), 75 - 94. https://doi.org/10.1016/j.obhdp.2013.12.005

\section{Historie}

Manuskript eingereicht: 12.10.2020

Manuskript nach Revision angenommen: 15.09.2021

Onlineveröffentlichung: 01.10.2021

\section{Förderung}

Diese Veröffentlichung wurde durch Sachbeihilfen des Landes Baden-Württemberg sowie der Pädagogischen Hochschulen Heidelberg und Ludwigsburg im Rahmen des Forschungs- und Nachwuchskollegs „Effektive Kompetenzdiagnose in der Lehrerbildung (EKoL)“ ermöglicht.

Open-Access-Veröffentlichung ermöglicht durch die Pädagogische Hochschule Heidelberg.

\section{Prof. Dr. Hendrik Lohse-Bossenz}

Pädagogische Hochschule Heidelberg

Keplerstraße 87

69120 Heidelberg

Deutschland

lohse@ph-heidelberg.de 